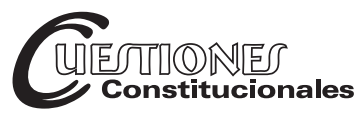

Revista Mexicana de Derecho Constitucional Núm. 41, julio-diciembre 2019

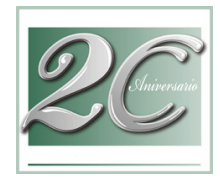

\title{
El derecho fundamental a la cultura física y al deporte: un derecho económico, social y cultural de reciente constitucionalización en México
}

\author{
The fundamental right to physical culture and sports: an economic, \\ social and cultural right of recent constitutionalization in Mexico
}

\section{Sonia VENEgAs ÁlVAREZ*}

El deporte tiene el poder de transformar al mundo. Tiene el poder de inspirar, de unir a la gente como pocas cosas.

\section{Nelson MANDELA}

RESUMEN: El derecho al deporte y la cultura física, reconocido con el carácter de fundamental por el ordenamiento jurídico mexicano en 2011, representa el primer eslabón de cara a la materialización del mismo en nuestro país. Se entiende como la prerrogativa a favor del gobernado para adquirir y desarrollar conocimientos relacionados con su cuerpo y los movimientos del mismo, así como a la práctica de actividades físicas sea por esparcimiento o de manera profesional al amparo del Estado, quedando obligado este último a la protección, al respeto y al fomento del mismo. Pero acaso lo relevante en la construcción de mecanismos de protección de este derecho fundamental es la comprensión del mismo, por un lado, en su vertiente genuina como derecho autónomo, y por otro, como mecanismo catalizador en la
ABSTRACT: The right to sport and physical culture recognized as fundamental by the Mexican legal in 2011 represents the first step in order to achieve the materialization in our country. The right to sport could be understand as the governed's prerogative to acquieve and develop knowledge related to his body and the movements thereof, as well as to practice of physical activities either for leisure or in a profesional manner under the protection of the State, which being obliged to the protection, respect and promotion of it. But perhaps, the most relevant issue is the construction of mechanisms to protect this fundamental right is its understanding: on the one hand in its genuine aspect as an autonomous right, in the other hand, as a catalyst procedure in the realization of other economic, social and cultural rights as healt,

* Profesora de tiempo completo en la Facultad de Derecho de la UNAM, México, miembro del SNI, directora del Seminario de Derecho Administrativo (turno vespertino) de la misma Facultad; svenegasa@derecho.unam.mx. 
concreción de otros derechos económicos, sociales y culturales, como la salud, la educación, etcétera, puesto que ello ha devenido en el diseño de políticas públicas (si bien sujetas a la temporalidad sexenal) permeadas de este nuevo enfoque, lo cual, sin duda, representa un avance por lo menos en el terreno jurídico.

Palabras clave: Deporte, educación física, actividad física, cultura física, derecho al deporte, derechos económicos, sociales y culturales. education etc. As a result, the manner in which the public policies are being designed has been permeated by this new approach.

Keywords: Sport, physical training, physical activity, right to sport, economic cultural and social rights.

SUMARIO: I. Introducción. II. Voces adyacentes y precisiones conceptuales. III. Fundamento constitucional del derecho a la cultura física y al deporte. IV. Contenido del derecho al deporte y la cultura física. V. El derecho al deporte y la cultura fisica: ordenamientos jurídicos que lo regulan. VI. Incidencia del derecho al deporte y a la cultura física. VII. Evaluación del derecho al deporte y a la cultura física en México. VIII. Conclusiones. IX. Bibliografía.

\section{INTRODUCCIÓN}

El derecho fundamental a la cultura física y al deporte es un derecho de reciente reconocimiento en el ordenamiento jurídico constitucional mexicano, contemplado en el artículo 4o. de la Constitución Política de los Estados Unidos Mexicanos, y representa un reto para naciones como la nuestra, con recursos limitados y un cada vez más trepidante expansionismo en la constitucionalización de derechos humanos; desentrañar el contenido de este derecho fundamental, y, en consecuencia, las acciones por tomar de cara a su cumplimiento son tareas de difícil realización. Y serán éstas las que ocupen los esfuerzos del autor, que busca abonar al debate actual no sólo en la delimitación del derecho, sino tratando de llevar el debate hacia las acciones gubernamentales, que muestran indicios alentadores en la construcción del derecho al deporte y la cultura física, los cuales se encuentran sujetos al imbatible enemigo: el sexenio, extraña unidad de medida de todo esfuerzo gubernamental. 


\section{VOCES ADYACENTES Y PRECISIONES CONCEPTUALES}

Abordar el tema del derecho fundamental a la cultura física y al deporte requiere el establecimiento de fronteras conceptuales entre términos usualmente confundidos: deporte, cultura física, actividad física y educación física, de modo tal que al hablar del contenido al derecho fundamental en cuenta, el lector se encuentre cierto de los alcances semánticos. Por un lado, puede efectuarse una diferenciación entre la cultura física, que comprende un conjunto de bienes, conocimientos, ideas, valores y elementos materiales que el hombre ha producido en relación con el movimiento y uso de su cuerpo, ${ }^{1}$ y por otro lado, la actividad física, que es la intención manifiesta en forma de movimiento del ser humano que relaciona su mente, cuerpo, emociones y necesidades de interacción con otros o con el entorno en el que habita; ${ }^{2}$ o bien, conforme a la Organización Mundial de la Salud, cualquier movimiento corporal producido por los músculos esqueléticos que exija gasto de energía, las cuales se llevan a cabo como parte de los momentos de juego, trabajo, formas de transporte activas, tareas domésticas y de actividades recreativas, ${ }^{3}$ mientras que la educación física es una parte de la educación que ofrece a los alumnos la oportunidad de tomar conciencia y compromiso frente a la actividad física de la totalidad de su cuerpo y el impacto de ésta en el ambiente social, ${ }^{4} \mathrm{o}$ bien, y hablando de la educación física de calidad, ésta es una experiencia de aprendizaje planificada, progresiva e inclusiva que forma parte del currículo en la educación infantil, primaria y secundaria, cuyo objetivo es ofrecer a niños y jóvenes experiencias adecuadas que les permitan adquirir las habilidades psicomotrices, la comprensión cognitiva de aptitudes sociales y emocionales que necesitan para llevar una vida físicamente activa. ${ }^{5}$

\footnotetext{
1 Artículo 3o. de la Ley General de Cultura Física y el Deporte.

2 Politica pública de deporte, recreación y actividad física para Bogotá 2009-2019, Bogotá, Alcaldía Mayor de Bogotá Secretaría de Cultura - Recreación y Deporte y Dirección de Culturas Recreativas y Deportivas, 2009, p. 29.

3 Organización Mundial de la Salud.

4 Traducción del autor del texto: Physical Education Curriculum Framework chapter I The nature of physical education, p. 2, disponible en: http://www.ed.gov.nl.caledu/ k12/curriculum/documents/physed/ch1.pdf.

5 Educación física de calidad. Guía para los responsables politicos, Organización de las Naciones Unidas para la Educación, la Ciencia y la Cultura, sector de ciencias sociales y humanas, 2015, p. 9.
} 
La problemática mayor descansa en definir y trazar los linderos del vocablo deporte. En el plano teórico y desde la perspectiva jurídica se ha planteado la imposibilidad de converger en una definición afín, puesto que este término no tiene, ciertamente, tradición en el campo jurídico, pero ante la imposibilidad de aprehenderse desde otras perspectivas de manera que sea asimilable por el derecho con una razonable certeza conceptual, se entiende preciso intentar acotarlo desde un planteamiento jurídico, puesto que el objetivo no es describir qué cosa es el deporte, sino de reconocerlo, de saber, con suficiente certidumbre, cuándo estamos frente a una de sus manifestaciones, de modo y manera que nos conste que a esa actividad le resulta aplicable el derecho al deporte. ${ }^{6}$ Precisado esto y a reserva de las observaciones que posteriormente se verterán, el deporte es una actividad física y mental de competición, expresada a través de diversas formas, institucionalizadas, sistematizadas y reglamentadas, que equilibra el desarrollo integral del ser humano, lo que potencialmente permite una justa convivencia y esparcimiento de los hombres en sociedad, y que por su trascendencia, en nuestros días, necesariamente es reconocida por el derecho. ${ }^{7}$ Puede decirse también que es toda aquella actividad física que, mediante una participación organizada, tenga por finalidad preservar y mejorar la salud física y mental, el desarrollo social, ético e intelectual con el logro de resultados en competiciones, ${ }^{8}$ y que de manera sucinta implica una actividad física, organizada, con una finalidad específica y competencia intrínseca. ${ }^{9}$

De tal forma que el deporte y actividad física si bien es cierto se encuentran íntimamente ligados, también lo es que distan mucho de ser sinónimos. El segundo atañe a cualquier movimiento motriz de nuestro cuerpo que implique un gasto en términos energéticos, y el deporte si bien conlleva en su propia naturaleza el movimiento del cuerpo humano, la forma organizada y planificada, así como la intención con la cual se lleva a cabo, es lo que le aporta la nota distintiva. De allí que muchos de los esfuerzos emprendidos hacia el entendimiento del deporte busquen la segmentación de esta actividad con base en la sujeción a determinadas reglas:

\footnotetext{
6 Real Ferrer, Gabriel, Derecho público del deporte, Alicante, Civitas, 1991, p. 96.

7 Del Carmen Clemente, Jalil Ascary et al., Derecho deportivo mexicano, México, Senado de la República-Asociación Mexicana de Investigación Jurídica Deportiva-Miguel Ángel Porrúa, 2008, p. 55.

8 Carta Iberoamericana del Deporte.

9 Hernández González, David, Derecho del deporte, Flores, México, 2014, p. 4.
} 
1) En un sentido amplísimo, el deporte abarcaría todas las manifestaciones o prácticas de ejercicio físico o físico intelectual del ser humano con o sin elementos auxiliares significativos o relevantes para tales actividades, de tipo materiales, con objetivos sanitarios, lúdicos o competitivos, en forma individual o de grupo, con o sin sumisión a reglas, de modo profesional, semiprofesional o puramente aficionado.

2) En sentido menos amplio serían los mismos elementos, pero incluyendo estructuras organizadas públicas y privadas, que oficializan, no toda pero si una parte muy significativa de aquel conjunto a partir del concepto de modalidades o especialidades.

3) En un sentido estricto, los elementos o componentes anteriores, pero con organizaciones públicas y privadas, con sujeción a reglas oficiales y en un plano exclusivamente competencial.

O bien, la posible diferenciación más simple:

- La educación física o deportiva.

- El deporte popular o para todos.

- El deporte élite o de alta competición.

- El deporte competición de carácter aficionado.

- El deporte profesionalizado. ${ }^{10}$

Entonces, un posible concepto de "deporte" es aquel que comprende aquellas actividades físicas institucionalizadas que supongan una superflua confrontación o competición consigo mismo o con un elemento externo. ${ }^{11}$ Puede además decirse que el vocablo "deporte" comprende todos aquellos juegos que son organizados bajo un sistema de reglas que establecen las modalidades de su desarrollo, previendo el empeño de habilidades y competencias, sean físicas o intelectuales, con un carácter competitivo. De modo tal que se excluyen de esta definición todas aquellas expresiones de juegos lúdicos, que si bien son actividades físicas e intelectuales, son prácticas cuya naturaleza esúnicamente motora. ${ }^{12}$

\footnotetext{
10 Bermejo Vera, José, Constitución y deporte, Madrid, 1998, p. 34.

11 Real Ferrer, Gabriel, op. cit., p. 96.

12 Coccia Massimo et al., Diritto dello sport, Florencia, La Monnier Università, 2004,
} p. 21. 
Nos encontramos entonces frente a conceptos que van desde la compresión de la totalidad de las actividades físicas hasta aquellas que se diferencian en función de la especialidad de la actividad física y la institucionalización de las mismas, postura que se manifiesta en la Ley General de Cultura Física y Deporte, al establecer una clara diferenciaciónentre el deporte social, de rendimiento y de alto rendimiento. El primero es, pues, tan sólo un factor de promoción, fomento y estímulo de participación de todas las personas en actividades deportivas con la finalidad de mejorar su salud, esparcimiento, educación; por el contrario, el deporte de rendimiento es aquel que promueve, fomenta y estimula que todas las personas mejoren su incursión en las actividades deportivas, pasando de la afición al alto rendimiento, o bien en la búsqueda de una relación laboral por práctica del deporte, y, por último, el deporte de alto rendimiento, que es la expresión de actividades físicas desarrolladas con altas exigencias técnicas y científicas de preparación y entrenamiento, lo cual le permite a los deportistas la participación en eventos internacionales de competencias. ${ }^{13}$

El deporte, entonces, presenta las características siguientes:

1) Es una actividad, al implicar una modificación del mundo exterior realizada por el propio ser humano, en forma individual o colectiva, con la utilización de sus fuerzas motrices.

2) La actividad es física y mental, al ser una combinación de la destreza, fortaleza y habilidad corporal del ser humano, guiada por la razón o el intelecto.

3) La actividad es de competencia, puesto que puede implicar marcas, tiempos, oposición o rivalidad para obtener logros.

4) La actividad se expresa de maneras diversas en nuestra realidad, reflejando los más variados gustos, técnicas, movimientos, hábitos, características físicas, entornos y climas.

5) La forma de la actividad es institucionalizada, sistematizada y reglamentada. El deporte tiene estas características toda vez que implica integrar una actividad con ciertos principios y orden para su desarrollo, así como para el alcance de sus fines u objetivos.

6) La actividad equilibra el desarrollo integral del ser humano. El deporte equilibra el desarrollo integral del ser humano permitién-

\footnotetext{
13 Artículo 5o. de la Ley General de Cultura Física y Deporte.
} 
dole un proceso cualitativo en el mejoramiento tanto físico como mental, y con ello calidad de vida.

7) La actividad puede lograr una justa convivencia y esparcimiento de los hombres en sociedad. El desarrollo del deporte lleva una justa relación de los hombres entre sí, pues permite su sana convivencia y esparcimiento y con ello su integración. ${ }^{14}$

Cumple, el deporte, las funciones que a continuación se enlistan:

1) Educativa. La actividad deportiva está considerada como un instrumento para equilibrar la formación y el desarrollo humano de la persona a cualquier edad. Los valores del deporte son el vehículo adecuado para desarrollar el conocimiento, la motivación, capacidades y disposición para el esfuerzo personal.

2) Mejora a la salud pública. La actividad física mejora la salud de los ciudadanos y contribuye a prevenir y luchar contra algunas enfermedades; asimismo, puede contribuir al mantenimiento de la buena salud y calidad de vida a una edad elevada.

3) Social. El deporte permite tener una sociedad más tolerante; éste puede facilitar la integración social y servir de apoyo al diálogo intercultural.

4) Lúdica. La actividad física es una parte importante del ocio y del entretenimiento individual y colectivo.

5) Cultural. La práctica deportiva permite al ciudadano arraigarse mejor en un territorio, conocerlo e integrarse mejor, y, con respecto al medio ambiente, buscar una mayor y mejor protección. ${ }^{15}$

Señalado lo anterior, el lector puede además diferenciar entre la cultura física - como conocimientos desarrollados por el ser humano acerca de su cuerpo y los movimientos de éste- y el deporte como ese conjunto de ejercicios efectuados por el ser humano, cuya institucionalización, perfec-

14 Hernández González, David et al., "Hacia la consolidación del derecho del deporte en México", Revista Académica de la Facultad de Derecho de la Universidad la Salle, México, núm. 11, año 6, julio de 2008, pp. 120 y ss.

15 Palomar Olmeda, Alberto, "El derecho del deporte y su forma de garantía en la sociedad del siglo XXI: una visión del derecho español”, en Montenegro González, Sadara y Hernández González, David (coords.), Justicia deportiva, México, Porrúa-Escuela Libre de Derecho, 2013, pp. 44-46. 
cionamiento y carácter competitivo le imprimen características, a partir de las cuales éste puede ser meramente recreativo y competitivo, basado, por supuesto, en pruebas y resultados.

\section{FUNDAMENTO CONSTITUCIONAL DEL DERECHO A LA CULTURA FÍSICA Y AL DEPORTE}

Será evidente para el lector la omisión en que se ha incurrido al pasar por alto - por lo menos hasta el momento - definir el contenido del derecho a la cultura física y el deporte; ello es así ya que este tema de vital importancia será objeto de estudio posteriormente; por el momento, la atención habrá de centrarse en el fundamento constitucional del mismo y sus antecedentes legislativos.

El decreto por el que se adiciona un párrafo al artículo cuarto y se reforma la fracción XXXIX- J del artículo 73 de la Constitución Política de los Estados Unidos Mexicanos, publicado en el Diario Oficial de la Federación el 12 de octubre de 2011, es la culminación del proceso legislativo iniciado el 28 de abril de 2008 en la Cámara de Senadores, con la presentación de la iniciativa con proyecto de decreto que reforma y adiciona los artículos 4o. y 73, en su fracción XXIX-J, de la Constitución Política de los Estados Unidos Mexicanos, a fin de reconocer la cultura física y el deporte como un derecho de todos los mexicanos, y cuya deliberación se sitúa en las Comisiones Unidas de Puntos Constitucionales, de Juventud y Deporte, y Estudios Legislativos.

Es precisamente este ir y venir legislativo el punto que nosotros habremos de tomar como partida en el desarrollo del tema que nos ocupa: la exposición de motivos de la iniciativa de reforma constitucional constituye, por un lado — el más obvio_-, la fuente de la cual podemos conocer las razones que llevaron al Constituyente a efectuar la modificación al texto constitucional, y, por el otro - el menos evidente-, un bosquejo del que será el enfoque gubernamental de la problemática por afrontar. De allí la necesaria cita textual de la exposición de motivos, en los términos siguientes:

La reciente constitucionalización del deporte no es un acontecimiento espontáneo, sino que responde a una evolución de los derechos y deberes públicos, íntimamente conectada con la transformación sucesiva de las tareas y cometidos de los poderes públicos frente a la sociedad... por 
lo que se debe formular la regulación de la cultura física y el deporte, previéndolos como parte importante de la política social y económica, buscando su reconocimiento constitucional, estableciendo en los poderes públicos su estímulo, fomento, protección y garantía de que la práctica del deporte y el acceso a la cultura física se dé en las mejores condiciones y que se favorezcan los valores humanos de la libertad, de la igualdad y de la solidaridad. Es por ello que los interesados en el tema insistimos en el reconocimiento expreso en la Ley Fundamental de la nación del derecho a esta importante actividad considerando que:

i. El reconocimiento expreso del derecho a la cultura física y el deporte en nuestra Constitución Política será un excelente cauce para la vertebración de las acciones públicas a favor del desarrollo de estas actividades.

ii. La existencia de estos preceptos, específicamente en nuestra Carta Magna, será una muestra del interés de los poderes públicos por la materia en general y no sólo por la actividad institucionalizada y organizada, resaltándose la importante labor del Estado respecto de su promoción y fomento.

iii. Con las reformas y adiciones propuestas no cabrán más posturas interpretativas unilaterales e incuestionables que desmienten, intervengan o interrumpan el desarrollo del ordenamiento jurídico en la materia.

iv. La estructura normativa de la cultura física y del deporte en nuestro país será reformada desde la perspectiva de ambas como un derecho y no como un concepto complementario o coyuntural, lo que permitirá la definición de una serie de directrices de actuación de los poderes público y las instancias particulares en sus respectivas y definidas competencias.

v. La adecuación y actualización de la fracción XXIX-J del artículo 73 permitirá la expedición de una nueva Ley Reglamentaria en la materia de la que podrán emanar un determinado grupo de reglamentos que complementen la legislación en materias como:

a) Deportistas de alto rendimiento;

b) Disciplina deportiva;

c) Dopaje;

d) Reconocimiento de la formación y titulación de los técnicos y entrenadores deportivos;

e) Prevención de la violencia en los eventos y espectáculos deportivos;

f) Registro y reconocimiento de asociaciones deportivas nacionales;

g) Conformación de delegaciones representativas nacionales, y

h) Regulación laboral especial de los deportistas profesionales, complementario de la Ley Federal del Trabajo. 
En virtud de lo anterior, cabe resaltar que el reconocimiento constitucional del derecho a la cultura física y el deporte, significará para los poderes del Estado la asunción definitiva de un compromiso encaminado al aseguramiento del bienestar social de nuestra sociedad, lo cual claramente reflejará el establecimiento de una nueva etapa en el desarrollo del Estado Mexicano... se trata de la inserción de la cultura física y el deporte en los ordenamientos jurídicos y aún más cuando se promueve su incorporación al derecho positivo mexicano desde su constitucionalización como un derecho social, cuestión sin duda alguna novedosa que directamente incidirá en nuestro contexto normativo y doctrinal. El derecho al deporte, aparece ligado a la concepción de lo que debe ser la actuación de los poderes públicos y el bienestar que éstos deben deparar a sus ciudadanos, el deporte, dotado de respaldo constitucional, se convierte en aspecto señero de la idea de calidad de vida que el Estado debe procurar a sus gobernados. Por lo tanto esta comisión dictaminadora, considera que la incorporación de las reformas propuestas, sin duda alguna enriquecerán el actual marco jurídico nacional y consagrarán la existencia en nuestro derecho positivo de una nueva materia inexistente en el país, como la del derecho público del deporte, permitiendo a nuestras comunidades universitarias, docentes e investigadores entre otras interesadas, a participar en el desarrollo jurídico de esta área de vital importancia para la vida nacional.

Hasta aquí son claros los motivos que han llevado al legislador mexicano a la constitucionalización del derecho a la cultura física y el deporte: la inserción en el texto jurídico de mayor envergadura en nuestro ordenamiento jurídico implica el reconocimiento del derecho a la cultura física y el deporte, entendido esto como el requisito indispensable para su concreción en la realidad. Sin embargo, la duda que emerge es cómo ese texto legal se proyectará en la vida diaria de los gobernados, la cual se disipa en la medida que se efectúa el análisis del segundo enfoque propuesto, y que implica entrever la estrategia dirigida al estímulo, fomento, protección y garantía del derecho a la cultura deportiva y el deporte, a través de la implementación de acciones públicas que tiendan a visibilizarlo como un derecho. Dicho de otro modo, la intervención del Estado deberá estar plenamente dirigida a la articulación de políticas públicas que proyecten el deporte y la cultura física como un derecho; veremos cómo ha sido esto. Y producto de este devenir histórico es que la redacción del artículo 4o. Constitucional en su parte conducente reza del modo siguiente: "Toda persona tiene derecho a la cultura física y a la práctica 
del deporte. Corresponde al Estado su promoción, fomento y estímulo conforme a las leyes en la materia".

Pero cabe cuestionarse cuál es la verdadera trascendencia de la inmersión del derecho al deporte y la cultura física en el ordenamiento jurídico mexicano a través de la previsión del mismo en la Constitución Política de los Estados Unidos Mexicanos, lo cual presupone que la reivindicación del deporte como un derecho humano no debe circunscribirse únicamente al marco de su consagración positiva en las Constituciones, pues se estaría relativizando el verdadero alcance del valor del mismo y desconociéndose que, en gran parte, se comenzó a reconocer por iniciativa de las organizaciones deportivas, gubernamentales o no, en cuyo seno se reconoció inicialmente el acceso a la práctica y conocimiento del deporte como valedero para el pleno desarrollo de la personalidad humana. Reconociendo ello, se está afirmando que estos derechos... significan un catálogo de exigencias que la consecución plena de la dignidad de la persona humana impone en la actualidad a toda organización jurídico-política, al igual que los derechos civiles y políticos, configurándose como plenos derechos subjetivos, en la medida en que se les valide tal condición en los respectivos ordenamientos jurídicos. Y su eficacia jurídica dependerá de las concretas posibilidades reales de ejercicio y protección jurídica con que cuenten dentro de los ordenamientos jurídicos a partir del reconocimiento extensivo que a los mismos se haga de los elementos caracterizadores de los derechos fundamentales. Así, en relación con el derecho al deporte, lo trascendental en la búsqueda de la eficacia y aplicabilidad directa y del debido control judicial, como muestra de aquéllos, será la determinación de los ejes mínimos de protección. ${ }^{16}$

\section{CONTENIDO DEL DERECHO AL DEPORTE Y LA CULTURA FÍSICA}

Este apartado tiene como finalidad desarrollar el contenido del derecho al deporte y a la cultura física; a partir de ello, analizaremos sus elementos y, por último, la trascendencia que el reconocimiento de este derecho por el ordenamiento constitucional tiene en el escenario jurídico mexicano. Esto último dará lugar al estudio de la naturaleza del derecho al deporte, entendido como un derecho de tipo económico, social y cultural, y las

16 Pachot Zambrana, Karel Luis, "El derecho constitucional al deporte en la doctrina y el derecho comparado", Cuestiones Constitucionales. Revista Mexicana de Derecho Constitucional, núm. 35, julio-diciembre de 2016, pp. 119 y ss. 
consecuencias que de esto se desprenden; esto último será la pauta introductoria al estudio de una peculiaridad del derecho que analizamos: no ha sido previsto en ningún instrumento internacional vinculante en materia de derechos fundamentales.

Una primera aproximación al contenido del derecho fundamental a la cultura física y deporte podría ser considerarlo como la prerrogativa que tiene toda persona a desarrollar actividades predominantemente físicas de manera individual o colectiva, con fines recreativos o competitivos, de forma preferentemente organizada y sujeta a normas para su desarrollo, las cuales son reconocidas en el entorno territorial en que se desarrollan como actividad deportiva. Esta definición no pretende señalar que este derecho es exclusivo de quienes se dedican de manera permanente o profesional al deporte, ni ocuparse sólo de actividades deportivas consideradas como olímpicas, ni sólo de aquellas actividades que cuenten con una organización nacional o internacional que las regule. El núcleo esencial de este derecho es la posibilidad que tiene una persona de desarrollar la actividad física (no comprendida como cualquier movimiento motriz del cuerpo humano) de su preferencia en el nivel o intensidad que comúnmente lo hace. ${ }^{17}$ Sus titulares podrán acceder a la práctica, preparación, conocimiento y competición de la modalidad o disciplina deportiva elegida, libre y voluntariamente, sin poder causar agravio ni lesión a los derechos o intereses legítimos de terceros, demandando una necesaria intervención pública el deporte aficionado, participativo, de masas, recreativo para todos, popular o de entretenimiento, donde concurran a su práctica las personas de modo voluntario, con la finalidad de contribuir a la integración en plenitud de una vida social, en la promoción de la salud, educación y preservación del medio ambiente. ${ }^{18}$ Esto, dicho de otro modo, le ofrece al titular la facultad de exigirle el acceso a la educación física y cultura física, la posibilidad de desarrollar bienestar y capacidades, sean estas físicas, psicológicas y sociales, por medio del deporte y la cultura física, a través de una oferta inclusiva y adaptada a las necesidades de todos los grupos sociales, cuya distinción pueda basarse en el sexo, la edad, la condición social y las aptitudes físicas, así como la participación en la toma y supervisión de decisiones en la materia.

17 Castilla Juárez, Karlos A., "El derecho humano a la práctica del deporte. Una propuesta desde y para la Constitución mexicana, Fair Play", Revista de Filosofía, Ética y Derecho del Deporte, Barcelona, bimestral, 2013, pp. 96 y ss.

18 Pachot Zambrana, Karel Luis, op. cit., pp. 119 y ss. 
No obstante todo lo anterior, una cuestión está pendiente: la relacionada con su carácter de derecho económico, social y cultural, ${ }^{19}$ esto es, la comprensión del derecho al deporte y a la cultura física como una especie de prerrogativa reconocida por el Estado relacionada con el lugar de trabajo, la seguridad social, la vida en familia, la participación en la vida cultural y el acceso a la vivienda, la alimentación, al agua, a la atención a la salud y a la educación, ${ }^{20}$ los cuales buscan garantizar condiciones materiales de vida digna para todos los ciudadanos, bajo el entendimiento de que dichas condiciones, además de su valor intrínseco, constituyen el presupuesto fáctico indispensable para el ejercicio efectivo de los restantes derechos por todos sus titulares, paralelamente a que la elevación del mínimo existencial que suponen hace posible el proceso de integración social que el Estado y la sociedad requieren para subsistir, constituyéndose como prerrequisitos para el disfrute de algunos derechos civiles y políticos. ${ }^{21}$

Estos derechos tienen como finalidad proporcionar el acceso de todos los ciudadanos a un mínimo vital indispensable, al bienestar entendido en su plena acepción, sin agotarse en sus contenidos meramente materiales, los cuales se viven frente al Estado y a través del Estado. ${ }^{22}$ De tal forma que al Estado le corresponde no únicamente procurar los medios materiales indispensables para el sostenimiento de los ciudadanos, sino también mejorar la calidad de vida. Esta actitud estatal garantiza la procura existencial, la satisfacción de las condiciones básicas de la existencia, lo que, entre otras medidas, exige al Estado realizar una serie de prestaciones sociales, por

19 Esta afirmación no ha sido del todo aceptada por los teóricos, puesto que la dificultad para entender el deporte como un derecho descansa en lo indeterminado de su naturaleza. Algunos teóricos lo sitúan como un derecho derivado de otros: su materialización implica derechos como el derecho de asociación, educación y a la vida cultural. Otros sostienen la independencia del mismo, aun y cuando para su ejercicio en plenitud necesite de la acción integrada o conjunta de otras libertades o derechos públicos.

20 "Preguntas frecuentes sobre los derechos económicos, sociales y culturales folleto informativo 33", Oficina del Alto Comisionado de las Naciones Unidas para los Derechos Humanos, p. 3.

21 Bazán, Víctor y Quesada, Luis Jimena, Derechos económicos, sociales y culturales cómo se protegen en América Latina y en Europa, Buenos Aires, Astrea, 2014, pp. 3 y ss.

22 La mención "a través del Estado" hace referencia al superado posicionamiento que pugnaba por la división de derechos fundamentales cuyo ejercicio suponía la abstención del Estado y en otros supuestos la acción del mismo. Sin embargo, y siguiendo a Cass R. Sunstein, en El costo de los derechos, ningún derecho podrá disfrutarse ante la inacción del Estado. Por tanto, la expresión "a través del Estado" hace referencia a la necesaria intervención del Estado como pre-condición indispensable en el disfrute de los mismos. 
lo que la intervención del Estado en materia deportiva resulta necesaria, dado el interés (nosotros acotaríamos obligación) de fomentar o promover la actividad deportiva: el deporte aparece entonces íntimamente ligado a la concepción de lo que debe ser la actuación de los poderes públicos y el bienestar que éstos deben deparar a sus ciudadanos; el deporte, dotado de respaldo constitucional, se convierte en aspecto señero de la idea de calidad de vida que el Estado debe procurar. ${ }^{23}$

Lo anterior es, más que elucubraciones teóricas, situar el derecho al deporte y a la cultura física como un derecho dentro del catálogo de los derechos económicos, sociales y culturales, lo que implica hacerle extensivo el catálogo de obligaciones derivadas de la suscripción por parte de México del Pacto Internacional de Derecho Económicos, Sociales y Culturales, las cuales habrán de desplegarse en la búsqueda de la materialización de los mismos. Tres son los tipos de obligaciones: respetar, proteger y realizar.

Las primeras buscan asegurar que el Estado se abstenga de interferir en el disfrute del derecho; proteger implica impedir que otras personas interfieran el disfrute del derecho, y realizar entraña la necesaria adopción de medidas apropiadas con miras a lograr la plena efectividad del derecho.

La adopción de medidas, específicamente las legislativas, tiene como finalidad el trascender hacia la adopción de políticas públicas que concreten los derechos económicos, sociales y culturales. De tal forma que al haberse efectuado el reconocimiento constitucional de los derechos lo que se busca es lograr un acceso justiciable de los mismos. Paralelamente, es necesario concientizarse acerca de la imposibilidad de lograr la plena efectividad de estas prerrogativas inmediatamente, y considerar que ésta se llevará a cabo progresivamente, por lo que la previsión de una flexibilidad necesaria que refleje las realidades del mundo real y las dificultades que implica para cada país asegurará la plena efectividad de los derechos económicos, sociales y culturales. Empero, el Comité concuerda en que a cada Estado le corresponde por lo menos satisfacer niveles mínimos o esenciales de aseguramiento de cada uno de los derechos. ${ }^{24}$ Ello se traduce en que al haberse reconocido en el texto constitucional el derecho a la cultura física y del deporte, se

\footnotetext{
23 Arnaldo Alcubilla, Enrique, El régimen constitucional del deporte, Madrid, Tecnos, 1992, pp. 35 y ss.

24 “Observaciones generales aprobadas por el Comité de Derechos Económicos, Social y Culturales núm. 2 Medidas internacionales de asistencia técnica y Preguntas frecuentes sobre los derechos económicos, sociales y culturales", Oficina del Alto Comisionados de las Naciones Unidas para los Derechos Humanos, folleto informativo núm.
} 
requiere un nuevo modelo de organización estatal, una transformación del Estado para que los poderes públicos se asuman como promotores eficaces de este derecho, teniéndose en cuenta que esta constitucionalización no se refiere sólo a la libertad de practicar deporte, sino de exigir una incorporación de todas las personas a la cultura física y la promoción de la práctica del deporte en sus diferentes manifestaciones. El derecho a la cultura física $\mathrm{y}$ al deporte es un derecho que conlleva prestaciones a cargo del Estado; implica el traspase de las decisiones sobre diseño de prioridades, de distribución y empleo de recursos del ámbito de la pura discrecionalidad política al ámbito del derecho. El gasto público destinado a la promoción, fomento y estímulo del deporte tendrá que tener una relación directa con el bien jurídico que requiere ser protegido. De manera tal que al haber establecido el legislador que toda persona tiene derecho a la cultura física y a la práctica del deporte, se debe entender que todos éstos estarán garantizados, por lo que resulta necesario establecer parámetros de actuación en función de cada subsistema de este derecho, las acciones a desarrollar por parte del Estado para satisfacer el derecho tutelado y, con base en esto, distribuir los recursos económicos suficientes para garantizarlos. ${ }^{25}$

Nos encontramos, entonces, frente a la necesidad de adoptar medidas que de manera paulatina tiendan a lograr la concreción de este derecho dentro de un plazo razonable, puesto que hemos superado ya la etapa de adopción de medidas legislativas - ya contamos con el sustento constitucional y el marco jurídico reglamentario-; los esfuerzos deben volcarse en la adopción - entre otras - de políticas públicas concretas encaminadas a hacer efectivo el derecho ya reconocido en nuestra Constitución. ${ }^{26}$ Por lo tanto, es dable concluir que es posible aseverar que el propósito fundamental de este derecho es asegurar el pleno desarrollo de la personalidad humana.

En este sentido, el derecho al deporte (y en nuestro país) y a la cultura física, comprendido como una manifestación de las personas que contribuye a configurar la gama de derechos inviolables del hombre, implica también

33, disponible en: http://www.ohchr.org/Documents/Issues/ESCR/FAQ\%20on\%20ESCRsp.pdf.

25 Flores Fernández, Zitlally, "El derecho a la cultura física y a la práctica del deporte en México. Su reconocimiento en México", Lex. Difusión y Análisis, cuarta época, año XVII, núm. 211, enero de 2013, pp. 55 y ss.

26 El lector puede tomar en consideración el contenido de la "Observación general núm. 3, la índole de las obligaciones de los Estados partes" del Comité de Derechos Económicos, Sociales y Culturales. 
el consecuente reconocimiento de la preexistencia del derecho a la práctica de las actividades deportivas respecto a la existencia del Estado. En esta sintonía, el derecho a la práctica de las actividades deportivas, entre los derechos inviolables del hombre, refiere:

- A la práctica del deporte desenvuelta en lo singular.

- A la práctica del deporte como un fenómeno interesante en la cual se ven inmiscuidos una pluralidad de individuos no organizados en una agrupación social, pero reunidos en virtud de la naturaleza misma de la actividad que se desarrolla.

- A la práctica del deporte desarrollada en organizaciones o agrupaciones estructuradas. ${ }^{27}$

\section{EL DERECHO AL DEPORTE Y LA CULTURA FÍSICA: ORDENAMIENTOS JURÍDICOS QUE LO REGULAN}

Tal y como lo hemos adelantado, el derecho al deporte y a la cultura física no se encuentra reconocido por ningún ordenamiento jurídico en materia de derechos humanos —en el sistema de protección americano-; ni la Convención Americana de Derechos, ni el Pacto Internacional de Derechos Económicos, Sociales y Culturales lo reconocen. Por el contrario, sólo la Carta Internacional de la Educación Física y el Deporte de 1978, adoptada en el seno de la Organización de las Naciones Unidas para la Educación, la Ciencia y la Cultura, reconoce en los términos siguientes este derecho:

Todo ser humano tiene el derecho fundamental de acceder a la educación física y al deporte, que son indispensables para el pleno desarrollo de su personalidad. El derecho a desarrollar facultades físicas, intelectuales y morales por medio de la educación física y el deporte deberá garantizarse tanto dentro del marco del sistema educativo como en el de los demás aspectos de la vida social. Cada cual de conformidad con la tradición deportiva de su país, debe gozar de todas las oportunidades de practicar la educación física y el deporte, de mejorar su condición física y de alcanzar el nivel de realización deportiva correspondiente a sus dones. Se han de ofrecer oportunidades especiales a los jóvenes, comprendidos los niños de edad preescolar, a las personas de

\footnotetext{
27 Coccia Massimo et al., op cit., p. 21.
} 
edad y a los deficientes, a fin de hacer posible el desarrollo integral de su personalidad gracias a un programa de educación física.

$\mathrm{Su}$ homólogo iberoamericano, la Carta Iberoamericana del Deporte, cuya adopción busca constreñir a los signatarios al fomento y difusión del deporte como factor fundamental de desarrollo integral del ser humano con el objetivo de:

Contribuir a que todos los sectores de la población tengan acceso a los beneficios de la práctica de la educación física, el deporte y la recreación, garantizando a todo los niños y los jóvenes la participación en planes y programas de educación física, deporte y recreación que desarrollen sus potencialidades físicas, morales, éticas e intelectuales, se asegure a todos la posibilidad de que practiquen el deporte y actividades físicas y recreativas, en un medio ambiente seguro y sano, desarrollando con la cooperación de organismos deportivos, programas prioritarios que favorezcan a niños y jóvenes, la mujer, la tercera edad, los discapacitados, campesinos, trabajadores y los pueblos indígenas, asegurando a todo deportista que posea condiciones para el alto rendimiento, la posibilidad de mejorar su capacidad competitiva y representar a su país en el concierto internacional. Proteger y salvaguardar las bases morales y éticas del deporte así como la dignidad humana y la seguridad de los que participan en actividades deportivas. Velar por la prevención del uso de sustancias prohibidas, esto dentro del concepto de educación permanente...

Considerar lo anterior es fundamental, puesto que la fuerza del derecho al deporte y la cultura física en el ordenamiento jurídico mexicano habría sido notablemente diferenciable de contemplarse dentro del catálogo de derechos previstos por el Pacto de Derechos Económicos, Sociales y Culturales en contraposición a las cartas del Deporte. Sin embargo, hacia nuestros días, esa distinción resulta irrelevante, puesto que existe ya un reconocimiento del carácter fundamental del derecho al deporte y la cultura física en México, lo cual ha dado lugar a la expedición de la ley reglamentaria del contenido del artículo 4o. constitucional. La Ley General de Cultura Física y Deporte es el cuerpo jurídico encargado de reglamentar el último párrafo del artículo 4o. constitucional, la cual se publicó en el Diario Oficial de la Federación el 7 de junio de $2013 .{ }^{28}$

28 El autor ha considerado oportuno resaltar el desfase temporal en que se incurrió al publicar este ordenamiento jurídico, puesto que, conforme al contenido del artículo segun- 


\section{INCIDENCIA DEL DERECHO AL DEPORTE Y A LA CULTURA FÍSICA}

Durante el desarrollo de este trabajo se ha hecho un fuerte hincapié en la interconexión del derecho fundamental al deporte y la cultura física en relación con otros derechos de la misma naturaleza; interdependencia entendida como condición necesaria para que el grado de concreción de otros derechos no se vea menoscabado, sin que ello derive en el entendimiento del derecho al deporte y a la cultura deportiva como una expresión, categoría o subdivisión de derechos fundamentales, tales como salud, educación o trabajo, puesto que ya hemos insistido en su naturaleza independiente y genuina. Por esto, el derecho al deporte es interdependiente, esto es, guarda estrechas relaciones con otros derechos, como son la vida, la salud, la integridad personal, la educación, mínimo vital y, en última instancia, con la dignidad de las personas. Es un derecho que no sólo incide en una dimensión individual, sino sobre todo colectiva, por el papel que puede tener para promover una cultura sana, valores, armonía, la autoestima y una relación armónica con los demás, esto es, una cultura de paz. Este derecho no sólo se desdobla en una dimensión del deporte de alto rendimiento, sino que implica la promoción de todas las prácticas deportivas, a todos niveles, desde los niños que tienen contacto con el sistema educativo nacional a nivel preescolar, pasando por todos los niveles educativos, implicando no sólo garantizar su acceso en las mismas condiciones, sino de promover a quienes se encuentran en una situación de rezago. ${ }^{29}$

Por ello, se parte del entendimiento del derecho al deporte y a la cultura física como la obligación a cargo del Estado de proporcionar las condiciones necesarias a los gobernados que permitan el acceso al oportuno desarrollo de actividades deportivas, así como al conocimiento acerca de las cualidades motrices de su cuerpo y los beneficios que el desempeño de movimientos constantes traen aparejados, el cual indiscutiblemente es un derecho fundamental, del cual conviene, sin embargo, precisar a cuál categoría pertenece, puesto que organismos internacionales, en el desempeño de sus laborales administrativas de los instrumentos en mate-

do transitorio del decreto de reforma constitucional publicado el 12 de octubre de 2011, la legislación reglamentaria debió de haberse expedido dentro del plazo de un año contado a partir de la publicación del propio decreto, situación que no ocurrió.

29 Lagarda Burton, Xóchitl, El derecho humano a la cultura física y el deporte, disponible en: http://coparmex.org.mx/el-derecho-humano-a-la-cultura-fisica-y-el-deporte/. 
ria de derechos fundamentales, distinguen las obligaciones inherentes a cada uno de éstos, y que no puede ser otra que la de derecho económico, social y cultural.

De lo anterior, y retomando lo ya anotado, se desprende que las políticas públicas, en el entendido que son una forma atomizada de decidir y ejecutar acciones de gobierno, representan la oportunidad idónea en el despliegue de la maquinaria institucional, que permita en efecto lograr los compromisos adquiridos, bajo el esquema de la constitucionalización del derecho en comento, la suscripción del Pacto Internacional de Derechos Económicos, Sociales y Culturales, y las no menos relevantes cartas del deporte, por lo cual el diseño de una estrategia específica deviene obligada. Para el autor no pasa desapercibida la existencia del sistema nacional de cultura física y deporte, que es una súper estructura a nivel federal cuya existencia se justifica en la medida en que las acciones de coordinación, seguimiento y evaluación de programas, acciones y procedimientos que formen parte de la ejecución de las políticas públicas que tengan por objeto, precisamente, el fomentar y estimular la cultura física y la práctica del deporte, deban seguir la pauta establecida. El sistema está conformado por una multiplicidad de instituciones del más alto nivel, que si bien dan respuesta a una de las formas en las cuales los Estados van cumplimentando los derechos económicos, sociales y culturales, lo cierto es, y desde la opinión del autor, que la forma atomizada y tanto más poderosa de incidir en la concretización de estos derechos es el diseño, implementación y evaluación de políticas publicas, por lo cual aun con la existencia de instituciones coordinadas sin programas específicos, no existe rumbo por transitar.

Una prueba de ese nuevo enfoque es la que el Programa Nacional de Cultura Física y Deporte 2014-2018 contempla, al identificar como una de las áreas de oportunidad del sistema deportivo mexicano la activación y el deporte como alternativas para el cuidado de la salud como prioridad, de manera que el deporte podrá ser entendido como una acción transversal que mitigue el escaso impacto y la ineficiencia que el mismo ha tenido en la sociedad, por lo cual se buscará erradicar el sedentarismo de la población. Para ello, se ha diseñado una estrategia que tienda a la incorporación del deporte a las políticas de salud con los objetivos siguientes:

- Ofrecer capacitación en materia de actividad física y salud a los médicos familiares y de atención primaria. 
- Impulsar la colaboración interinstitucional con los institutos de seguridad social y deporte municipal y estatal en conjunto con la Secretaría de la Salud.

- Crear una red de centros homologados de medicina deportiva y reconocimientos médicos-deportivos oficiales. ${ }^{30}$

Pero dicha perspectiva había sido ya abordada a nivel internacional con anterioridad en el seno de la Organización Mundial de la Salud, al reconocer a la actividad física como un factor de crucial importancia en el mejoramiento de la salud, puesto que la Estrategia Mundial sobre Régimen Alimentario, Actividad Física y Salud de 2004 establece un estudio integral acerca de la necesidad de insertar el enfoque de la salud y la actividad física con miras a diseñar e implementar políticas públicas que centren el deporte como un medio de prevención y reducción de enfermedades no contagiosas. Este enfoque de 2004 encuentra un eco mayor en el documento denominado Recomendaciones Mundiales sobre Actividad Física para la Salud de 2010, el cual insta a los gobiernos de cara a la adopción de medidas que centren a la actividad física y el deporte como pilares sobre los cuales se fundamenten las políticas nacionales y la intervención de la salud pública; el establecimiento de metas y objetivos por alcanzar en materia de promoción de la actividad nacional en la totalidad del territorio de los Estados parte; al fomento de la participación intersectorial; la asignación de recursos monetarios que permitan materializar dicho derecho, y la facilitación de mecanismos que vigilen y controlen la evolución de la actividad física en la población. ${ }^{31}$

\section{EVALUACIÓN DEL DERECHO AL DEPORTE Y A LA CULTURA FÍSICA EN MÉXICO}

Partiendo de lo anterior, válidamente puede cuestionarse el lector cómo vamos en México en la materialización de este derecho al deporte y a la cultura física a casi siete años de su constitucionalización. Para tratar de responder ese cuestionamiento debe recordarse que es compromiso del Estado la intervención para su estímulo, fomento, protección y garantía del derecho a

\footnotetext{
30 Programa Nacional de Cultura Física y Deporte 2014-2018.

31 Recomendaciones mundiales sobre actividad física para la salud, Organización Mundial de la Salud, 2010, p. 11.
} 
la cultura deportiva por medio de acciones públicas, las cuales, en primera instancia, encuentran fundamento en la Ley General de Cultura Física y Deporte, y decimos en primera instancia ya que de ésta se derivan algunos otros documentos de relevancia, tal como el Plan Nacional de Desarrollo o el Programa Nacional de Cultura Física y Deporte, que en un análisis conjunto muestran el grado de concreción - por lo menos en estrategiadel derecho a la cultura física y deporte en México, por lo cual conviene detenerse en su estudio. La mayor relevancia de la Ley General de Cultura Física y Deporte estriba en el establecimiento de directrices que guían el procedimiento de promoción, fomento y estímulo de la cultura física y el deporte y que consisten en las siguientes actividades:

1) Difusión del contenido y valores comprendidos por la cultura física y deportiva.

2) Promoción, fomento y estímulo de actividades relacionadas con competencias deportivas.

3) Promoción, fomento y estímulo de investigaciones sobre la cultura física.

4) Promoción, fomento y estímulo a nivel nacional de una cultura deportiva que haga del deporte un bien social y un hábito.

5) Difusión del patrimonio cultural deportivo.

6) El otorgamiento de apoyos en dinero o especie, capacitación, asesoría, asistencia y gestoría por las autoridades competentes.

7) La construcción, remodelación, ampliación, adecuación, mantenimiento, conservación y recuperación de instalaciones para la atención de la demanda social.

8) Promoción, coordinación e impulso de la enseñanza, investigación, difusión del desarrollo tecnológico, aplicación de conocimientos científicos relacionados con la activación física, cultura física y deporte, construcción de centros de enseñanza y capacitación de actividades.

Adicionalmente, reconoce el texto legal la necesidad de contar con el respaldo de la totalidad de las instituciones gubernamentales, deportivas y educativas en el acceso a la educación física, actividad física y deporte, debiendo asignársele un lugar preponderante dentro del sistema educativo, particularmente a nivel primaria y secundaria, en los cuales el desarrollo de estas actividades debe efectuarse de manera cotidiana en la vida de 
niños y jóvenes. Si bien es cierto esta ley reglamentaria se encuentra un paso adelante de la mera existencia de legislación, al contemplar acciones concretas, lo cierto es que retoma dicho enfoque al precisar la obligación a cargo de las autoridades públicas y entidades inmiscuidas en el estímulo y promoción del deporte de elaborar y aplicar leyes y reglamentos, definir planes nacionales de desarrollo del deporte con objetivos claros, y adoptar todas las demás medidas de estímulo de la educación física, la actividad física y el deporte, comprendiéndose la prestación de asistencia material, financiera y técnica, y abordando un punto fundamental en materia de derechos fundamentales, no olvida acentuar la relevancia de la inversión constante a cargo del Estado como muestra del compromiso con la causa, a través del necesario incremento en las asignaciones presupuestarias para la oferta pública de programas de educación física de calidad. Por último, se recalca la obligatoriedad de mantener y proporcionar espacios, instalaciones, equipos y opciones indumentarias adecuados y seguros; proporcionar y optimizar instalaciones, servicios y equipos destinados a la causa, teniéndose en cuenta las posibilidades y condiciones que ofrece el entorno natural, la planificación urbana, rural y del transporte.

Este es el panorama de la Ley General de Cultura Física y Deporte, pero tal y como lo habíamos señalado, de ella derivan cuestiones de relevancia tal que han de quedar plasmadas en el documento que guía el desarrollo de la administración pública de manera sexenal: el Plan Nacional de Desarrollo. Por lo tanto, y a partir de 2013, en la planeación nacional debe tomarse en consideración el desarrollo de la cultura física y el deporte. ${ }^{32}$ Aquí conviene hacer una pausa. Si se traza una línea del tiempo, la reforma constitucional se sitúa al final del sexenio comprendido de 2006 a 2012, esto es, hacia 2011, a poco menos de un año de concluir el mandato de la administración federal quedaba poco margen de actuación institucional; no obstante lo anterior, el Plan Nacional de Desarrollo ya preveía el diagnóstico y plan de acción en la inclusión del deporte en la agenda pública.

El Plan Nacional de Desarrollo 2007-2013 se asienta en cinco ejes: i) Estado de derecho y seguridad; ii) economía competitiva y generadora de empleos; iii) igualdad de oportunidades; iv) sustentabilidad ambiental, y v) democracia efectiva y política exterior responsable, pero acaso a nosotros nos resulta útil sólo el tercer eje: igualdad de oportunidades, que consiste en apuntar a la igualdad de oportunidades como promotor del desarrollo y

\footnotetext{
32 Conforme al artículo 9o. de la Ley General de Cultura Física y Deporte.
} 
bienestar de los mexicanos, por lo cual debe figurar como un objetivo de la política social.

Este tercer eje, a su vez, se haya atomizado en diversos apartados, de los cuales superación a la pobreza y cultura, arte, recreación y deporte contienen las estrategias por llevar a cabo en el impulso del deporte, por lo que su análisis resulta obligado.

El eje "3.1 superación de la pobreza" busca consolidar políticas públicas que continúen fortaleciendo el acceso a servicios básicos, y al mismo tiempo coordinar la política social y económica para elevar el ingreso y el empleo de la población en general, especialmente de aquella que se desenvuelve en condiciones precarias. Para su consecución, el objetivo 12 propone la impartición de educación integral en la totalidad del sistema educativo, la cual, para ser completa, debe abordar junto con las habilidades para aprender, aplicar y desarrollar conocimientos, el aprecio por los valores éticos, el civismo, la historia, el arte y la cultura, idiomas y la práctica del deporte, eliminándose la diferencia central entre el sistema de educación público frente al privado, que es precisamente dicho enfoque integral.

De allí que la estrategia 12.8 obligue a la totalidad de los planteles escolares a la inclusión de las actividades físicas y el deporte dentro del sistema educativo, con el consecuente abatimiento de la obesidad y el sedentarismo, contribuyéndose a la mejor de las condiciones de vida de los mexicanos.

El segundo eje, "3.8 cultura, arte, recreación y deporte", al buscar el fortalecimiento de las manifestaciones culturales de México, presta especial atención a las políticas públicas que incidan en el arte, el deporte y la recreación, al considerarlas actividades centrales para la salud y vitalidad de la sociedad, impulsándose también la práctica del deporte en sus diversas manifestaciones para que más mexicanos se ejerciten y se superen a sí mismos. Todo ello queda plasmado en el objetivo 23, cuya finalidad es el fomento a la cultura de la recreación física, promotora de la realización de algún ejercicio físico o deporte por la totalidad de los mexicanos; reflejado en la estrategia 23.1, la formación y consolidación de una cultura deportiva entre todos los grupos sociales y de edad, y en todas las regiones del país, y la estrategia 23.2, enfocada al desarrollo de infraestructura deportiva y maximización de los espacios públicos abiertos dedicados a la promoción de la práctica de deportes, mientras se alienta la construcción de infraestructura deportiva y se procura la multimodalidad de la misma.

El siguiente esquema de trabajo sexenal propuso la persecución de cinco pilares: i) México en paz; ii) México incluyente; iii) México con educación 
de calidad; $i v)$ México próspero, y v) México con responsabilidad global. Desarrollado en una especie de acto reflejo, es el tercer eje el que contiene lo relativo al deporte y la cultura física. Este plan sexenal de trabajo percibe al deporte como un pilar esencial para contar con una sociedad saludable y con cohesión social. El impulso a esta actividad se concibe como un fin en sí mismo, con implicaciones positivas en todos los aspectos de la sociedad, motivo por el cual se ha transformado en un objetivo fundamental del gobierno federal en curso; por ello se consideró necesario el desarrollo del talento deportivo en la juventud para promover una cultura de la salud; ${ }^{33} \mathrm{a}$ través del "Plan de acción III.2. Articular la educación, la ciencia y el desarrollo tecnológico para lograr una sociedad más justa y próspera", el cual, en la búsqueda de la promoción del deporte de manera incluyente para fomentar una cultura de salud, se ha propuesto impulsar que la mayoría de la población tenga acceso a la práctica de actividades físicas y deportivas en instalaciones adecuadas, con la asesoría de personal capacitado. Asimismo, se debe promover el aprovechamiento total de la infraestructura deportiva nacional existente, recuperar espacios públicos para la actividad física y garantizar la adecuada planeación de la infraestructura del sector. Todo lo anterior fue plasmado en la estrategia de acción 3.4.1 del Plan Nacional de Desarrollo: crear un programa de infraestructura deportiva y que consiste en: i) contar con información confiable, suficiente y válida de las instalaciones existentes a nivel municipal, estatal y federal, para conocer el estado físico y operativo de las mismas; ii) definir con certeza las necesidades de adecuación, remodelación y óptima operación de las instalaciones deportivas, incluyendo las escolares; iii) recuperar espacios existentes y brindar la adecuada respuesta a las necesidades futuras que requieren los programas deportivos; $i v$ ) poner en operación el sistema de evaluación en materia deportiva que garantice la eficiencia de los recursos financieros invertidos en el deporte nacional. Paralelamente, la estrategia 3.4.2. Diseñar programas de actividades físicas y deporte diferenciados para atender las diversas necesidades de la población propuso la creación de un programa de actividad física y deporte tendente a la disminución de los índices de sobrepeso y obesidad; facilitar la práctica deportiva sin fines selectivos, con un enfoque que promueva la adquisición de valores para el trabajo en equipo, respeto a

33 Con base en el contenido del Plan Nacional de Desarrollo, hacia 2012 se contaba con 1,200 espacios activos que prestan servicios a más de 700,00 habitantes de manera mensual, y alrededor de 4,900 centros del deporte escolar y municipal que atienden a 12 millones de niños, jóvenes y adultos, sin que la demanda esté aún cubierta. 
las reglas y obtención del éxito mediante el esfuerzo individual y colectivo; estructurar con claridad dos grandes vertientes para la práctica de actividades deportivas: deporte social y deporte de representación, y facilitar el acceso a la población con talentos específicos al deporte estrictamente selectivo.

Por último, y quizá el más aterrizado esquema de trabajo, es el Programa Nacional de Cultura Física y Deporte 2014-2018, cuyo sustento se encuentra asimismo en la Ley General de Cultura Física y Deporte, que condensa los objetivos, estrategias y líneas de acción que buscan incorporar, en el modo de vida cotidiano, la actividad física y deportiva, vista como un mecanismo de mejora en la calidad de vida, el fomento a la salud y la sana convivencia por medio de la consecución de 5 objetivos disgregados en sendas estrategias. De esas 5 estrategias, sólo tres nos resultan útiles para los efectos de este trabajo: i) "Aumento en la práctica del deporte social para mejorar el bienestar social de la población"; ii) "Mejorar la infraestructura deportiva del país al garantizar la óptima operación tanto de la ya existente, como de aquella que sea de nueva creación para acercar la práctica del deporte al ciudadano"; iii) "Impulsar la gestión del deporte desde una perspectiva transversal para incidir en la vida de las personas". La primera de éstas es un esquema que busca potencializar la práctica del deporte desde la unidad de división territorial más pequeña en México: el municipio, ello por medio de la formación de esquemas deportivos y capacitación técnica; "mejorar la infraestructura deportiva del país al garantizar la óptima operación tanto de la ya existente, como de aquella que sea de nueva creación para acercar la práctica del deporte al ciudadano"; es un área de oportunidad que busca incrementar y aprovechar la infraestructura deportiva, por lo que el énfasis debe aplicarse no sólo en el aumento cuantitativo de las instalaciones, sino también en el cualitativo. Y es aquí donde se muestra la necesaria incursión de la inversión privada a la par del sector público, de manera tal que se dinamice el crecimiento tanto en la construcción como en la gestión de centros deportivos y de uso social y comunitario, y, por último, "Impulsar la gestión del deporte desde una perspectiva transversal para incidir en la vida de las personas", es probablemente el objetivo de mayor envergadura e impacto, puesto que incide en el factor humano o social de nuestro país, al intentar mutar la concepción del deporte como un hecho aislado sin proyección o repercusión en otras áreas de la vida. Se trata, pues, de mostrarlo como un factor de cambio social: en lo educativo, en la salud, en el empleo, en lo económico y como factor de cohesión social. 
Una vez que el lector conoce en su amplio espectro el derecho al deporte y a la cultura física en México, sólo resta a modo de propuesta abordar la actuación del órgano de la administración pública federal encargado de ejecutar lo relacionado con la materia: la Comisión Nacional de Cultura Física y Deporte, misma que conforme a la Ley General de Cultura y Deporte es un organismo descentralizado que cuenta con personalidad jurídica y patrimonio propio y que medularmente tiene el deber de proponer, dirigir, ejecutar, evaluar y vigilar la política nacional relacionada con la cultura física y el deporte, de la cual debemos señalar su necesaria reformulación orgánica en aras de la construcción de un garante real del derecho humano al deporte y a la cultura física.

Este es pues el panorama del derecho al deporte y la cultura física en México. Como el lector podrá apreciar, existe voluntad política en transitar hacia un diseño mucho más estructurado en materia de planeación de las acciones que darán paso del texto jurídico a la realidad social de las prerrogativas reconocidas por el ordenamiento jurídico mexicano. Pero nuestra crítica pudiera efectuarse hacia el tinte inminentemente político de las mismas, y es que al igual que acontece en muchos otros aspectos, las rutas, programas, iniciativas, instrumentos y diseños institucionales que se encuentran son de corte sexenal, y es por todos reconocido que ese término perentorio acota el impacto de cualquier política pública, de tal forma que los recursos vertidos y proyecciones efectuadas quedan truncas en su aplicación, sujetas siempre al vaivén del titular del Ejecutivo Federal, cuya tendencia es lamentablemente denostativa hacia lo hecho en el pasado, por lo cual sería interesante considerar la posibilidad de abrir tópicos cuya relevancia en la social sea tal que su diseño e implementación no quede sujeto a lo meramente sexenal, entre los cuales, ciertamente, el deporte y la cultura física habrán de ubicarse.

Al margen de lo anterior, aunque vinculado, aparece el tema de la sinergia entre el gobierno como agente activo en la ardua tarea de establecer las condiciones que permitan el disfrute de estos derechos, en relación con el sector privado. Tópico que la Carta Iberoamericana del Deporte contempla al establecer: "Para la realización de los objetivos de la presente carta es esencial una estrecha relación entre las organizaciones deportivas gubernamentales y no gubernamentales...", y que se encuentra replicada en la legislación nacional, ya que la Ley General de Cultura Física y Deporte reconoce la necesaria incursión de los entes privados en los términos siguientes: 
El sistema deportivo mexicano en sus diferentes niveles de práctica y en los distintos ámbitos territoriales de actuación, necesita de una adecuada colaboración con el sector privado para posibilitar un impulso en la aplicación de inversiones y recursos que permita explorar nuevos horizontes. Los diferentes tipos de gestión en la aplicación de los principios de colaboración con el sector privado, representan una oportunidad para detonar inversiones con la construcción de instalaciones deportivas y la generación de nuevas áreas de negocio que complementen la oferta pública deportiva.

Nótese que la colaboración no sólo descansa en el diseño o implementación de acciones, sino que es preponderantemente económica; el papel del sector privado, a partir de la lectura integral de la ley general, busca incentivar la participación de los privados en la aportación de recursos monetarios que, en conjunto con lo presupuestado por el Estado, permitan potenciar el impacto de las inversiones, sin que por ello en estos entes coadyuvantes pueda descansar la obligación de dotar al gobernado de las prestaciones que el derecho al deporte y cultura física entrañan.

\section{CONCLUSIONES}

Actividad física, cultura física y deporte son tres conceptos cuyo adecuado manejo garantizan el satisfactorio entendimiento del tema aquí planteado; la distinción estriba en que mientras la actividad física hace referencia a cualquier movimiento motriz, en el deporte, si bien abraza dichos movimientos, la intención con la cual se efectúan, así como la organización e institucionalización de los mismos, permiten su diferenciación. Por último, la cultura física es todo ese bagaje de conocimientos que orbitan en torno a los movimientos que con nuestro cuerpo podemos llevar a cabo.

El carácter de fundamental del derecho al deporte y la cultura física no puede ya discutirse; apelando tan sólo a su ubicación en el ordenamiento jurídico mexicano, el encontrarse previsto por la norma fundamental la discusión queda, para efectos prácticos, zanjada. Podría también argüirse que la simple mención del derecho por la Constitución no le imprime el carácter de fundamental, y es cierto. Su relevancia para el desarrollo de la vida humana, sus bondades en el desarrollo social son tales que son éstas las características que le otorgan el carácter de derecho humano, cuyo reconocimiento por el constituyente a nivel constitucional, lo tildan de derecho fundamental. 
El reto se presenta ahora en la determinación de su naturaleza, que no es otra que la de un derecho económico, social y cultural, situación que no es mínima, ya que a partir de ésta se derivan una serie de obligaciones que el Estado mexicano debe afrontar en la búsqueda del cumplimiento de compromisos internacionales, entre las cuales la adopción de políticas públicas que fomenten, promuevan y garanticen la posibilidad de acceder, por un lado, al desarrollo de actividades motrices que abonen a la salud, desarrollo integral de los gobernados y fomenten la cohesión social, así como la adquisición de conocimientos acerca del tema, se ciernen como uno de los muchos caminos que permitirán la materialización de este derecho en México.

La tarea de dotar de esas condiciones mínimas a los gobernados, que les permitan acceder a la práctica del deporte y el bagaje de conocimientos sobre la materia conocidos como cultura física, no es ni puede ser una empresa propia del gobierno, sino que requiere, como en otras tantas áreas, apoyo y participación del agente privado. Ésta es pues una muestra de la clara interconexión de los agentes sociales que convergen en la creación, pero también en el funcionamiento del Estado.

\section{BIBLIOGRAFÍA}

ARnaldo Alcubilla, Enrique et al., El régimen constitucional del deporte, Madrid, Tecnos, 1992.

BAZÁN, Víctor y QUESADA, Luis Jimena, Derechos económicos, sociales y culturales cómo se protegen en América Latina y en Europa, Buenos Aires, Astrea, 2014.

Bermejo Vera, José, Constitución y deporte, Madrid, 1998.

BERMEJO VerA, José, "Derecho al deporte. Los derechos fundamentales en el deporte", en GonzÁlez Montenegro, Sadara y Hernández GonZÁleZ, David (coords.), Obra jurídica enciclopédica. Derecho del deporte, México, Porrúa-Centro de Investigación e Informática Jurídica, 2013.

Carmen Clemente, Jalil Ascary del et al., Derecho deportivo mexicano, Senado de la Republica, LX Legislatura-Asociación Mexicana de Investigación Jurídica Deportiva-Miguel Ángel Porrúa, Librero, 2008. 
CASTILla JuÁreZ, Karlos A., "El derecho humano a la práctica del deporte. Una propuesta desde y para la Constitución mexicana, Fair Play", Revista de Filosofí, Ética y Derecho del Deporte, Barcelona, bimestral, 2013.

Coccia, Massimo et al., Diritto dello sport, Florencia, La Monnier Università, 2004.

DASSIE, Roberta, "I diritti umani e lo sport", Revista elettronica del Centro di Documentazione Europea dell' università Kore di Enna.

Educación física de calidad. Guía para los responsables políticos, Organización de las Naciones Unidas para la Educación la Ciencia y la Cultura, sector de ciencias sociales y humanas, 2015.

FLORES FERNÁNDEZ, Zitlally, "El derecho a la cultura física y a la práctica del deporte en México. Su reconocimiento en México", Lex. Difusión y Análisis, cuarta época, año XVII, núm. 211, enero de 2013.

HERnÁndez GonZÁLEZ, David, Derecho del deporte, México, Flores, 2014.

HERnÁNDEZ GonZÁLEZ, David et al., "Hacia la consolidación del derecho del deporte en México", Revista Académica de la Facultad de Derecho de la Universidad la Salle, México, núm. 11, año 6, julio de 2008.

LAGARDA BURTON, Xóchitl, El derecho humano a la cultura fisica y el deporte, http://coparmex.org.mx/el-derecho-humano-a-la-cultura-fisicay-el-deporte/.

MANIATIS, Antonio, "El derecho al deporte", Anuario da Facultade de Dereito da Universidade da Coruña, vol. 2 , 2017.

MANIATIS, Antonio, "La funzione sociale dello sport e il dialogo interculturale nel sistema comunitario", Rivista italiana de diritto pubblico comunitaria, Milano, año XIX, núm. 2, bimestral, 2009.

“Observaciones generales aprobadas por el Comité de Derechos Económicos, Social y Culturales núm. 2 Medidas internacionales de asistencia técnica y Preguntas frecuentes sobre los Derechos Económicos, Sociales y Culturales", Oficina del Alto Comisionado de las Naciones Unidas para los Derechos Humanos, folleto informativo, núm. 33, disponible en http://www.ohchr.org/Documents/Issues/ESCR/FAQ\%20on\%20ESCRsp.pdf.

PACHOt ZAmbranA, Karel Luis, "El derecho constitucional al deporte en la doctrina y el derecho comparado", Cuestiones Constitucionales. Revista Mexicana de Derecho Constitucional, núm. 35, julio-diciembre de 2016. 
PAlOMAR OlmedA, Alberto, "El derecho del deporte y su forma de garantía en la sociedad del siglo XXI: una visión del derecho español", en Montenegro GonzÁlez, Sadara y Hernández GonZÁlez, David (coords.), Justicia deportiva, México, Porrúa-Escuela Libre de Derecho, 2013.

Physical Education Curriculum Framework chapter I The nature of physical education, http://www.ed.gov.nl.ca/edu/k12/curriculum/documents/ physed/ch1.pdf.

Política Pública de Deporte, Recreación y Actividad Física para Bogotá 2009-2019, Bogotá, Alcaldía Mayor de Bogotá Secretaría de CulturaRecreación y Deporte y Dirección de Culturas Recreativas y Deportivas, 2009.

"Preguntas frecuentes sobre los derechos económicos, sociales y culturales folleto informativo 33", Oficina del Alto Comisionado de las Naciones Unidas para los Derechos Humanos.

ReAl Ferrer, Gabriel, Derecho público del deporte, Alicante, Civitas, 1991.

Solar CuBILlas, Luis Vicente, "El «deporte para todos», cuestión de Estado. El deporte para todo en Europa y en España", Revista Española de Educación y Deportes -REEFD-, España, año LXVII, núm. 409, 20. trimestre, 2015.

STELITANo, Antonellla, Il diritto allo sport: evoluzione del concetto di diritto allo sport negli atti internazionali rilevanti, disponible en: https:// www.iissbojano.gov.it/attachments/article/90/09_Stelitano.pdf.

Fecha de recepción: 07 de junio de 2018.

Fecha de aceptación: 21 de octubre de 2018. 